\title{
In silico Discovery of anti-SARS-CoV-2 agents via docking screening and ADMET properties
}

Hanine Hadni ( $\square$ hadni.hanine@yahoo.fr)

Faculty of Sciences Dhar El mahraz https://orcid.org/0000-0002-4952-9533

Menana Elhallaoui

Faculty of Sciences Dhar El mahraz

\section{Research Article}

Keywords: SARS-CoV-2, COVID-19, Molecular docking, hybrid molecules, ADMET properties, Druglikeness

Posted Date: July 6th, 2020

DOI: https://doi.org/10.21203/rs.3.rs-37947/v1

License: () (7) This work is licensed under a Creative Commons Attribution 4.0 International License. Read Full License 


\section{Abstract}

The novel coronavirus (SARS-CoV-2) is the causative factor in the COVID-19 pandemic that infected more than 3 million people and the number of deaths continues to rise. There are currently no drugs or vaccines available to treat this disease, which is a serious global health problem. In this study, we performed molecular docking of heterocyclic molecules based on Quinoline, Triazine and Azaaurone, and to increase their inhibitory efficacy, we have used hybrid molecules linking two pharmacophores such as quinoline-oxalamide, quinoline-triazine and azaaurone-triazine. In order to accelerate the drug discovery process against this new SARS-CoV-2. Hydroxychloroquine and Azithromycin drugs showed anti-SARS-CoV-2 activity, which is why we have considered these drugs as a reference for other molecules. Most compounds showed high inhibitory activity against SARS-CoV-2, and promising results in ADMET properties and drug likeness. Thus, these compounds present excellent drug candidates. These results would be of great help in leading discovery and optimization for new drug against novel coronavirus.

\section{Introduction}

Coronaviruses are a large family of viruses that affect several animal species and some of these viruses can also affect humans. The pathologies they cause are varied but they mainly affect the respiratory and digestive systems [1-3]. Studies have found that six strains of coronavirus are capable of infecting humans, four strains of coronavirus circulating each year to cause the common cold, and two other strains of the infamous infectious coronavirus are Severe Acute Respiratory Syndrome (SARS-CoV) and Middle Eastern Respiratory Syndrome (MERS-CoV) [4,5], which have infected more than 10,000 people globally in the last two decades, with high mortality rates (9.6\% for SARS-CoV and $34.4 \%$ for MERS-CoV) [6,7].

In December 2019, the world is facing the same situation as the previous epidemic due to a new coronavirus, which has been named Coronavirus Disease 2019 (COVID-19) caused Severe Acute Respiratory Syndrome Coronavirus 2 (SARS-CoV-2), with symptoms including fever, dyspnea, asthenia and pneumonia [8,9]. The current mortality rate of COVID-19 remains lower than SARS-CoV or MERS-CoV [10], however, COVID-19 is highly contagious it spreads in a frightening way more than 3 million people with COVID-19 with more than 250000 deaths in 204 countries in just 5 months [11]. Unfortunately, there is no effective treatment at the beginning of the outbreak to prevent its spread [12]. Current development of drugs is very slow, because it takes several years for a molecule of therapeutic interest to become a new drug on the market. This is why it has become extremely urgent to speed up considerably the development of new drugs that can cure patients with COVID-19.

The use of molecular modeling such as molecular docking has provided very impressive results in the domain of drug design in the last years [13]. In this regard, we performed molecular docking of 30 heterocyclic molecules (Fig. 1) to orient experimental chemists towards molecules with anti-SARS-CoV2 potential. These proposed heterocyclic molecules were inspired by compounds with antimalarial potential. Quinoline and its derivatives have attracted great interest from medical chemists because it has several reaction sites and is one of the most important pharmacophores in the fight against malaria [14,15]. azaaurone are very important therapeutic targets, as they show several biological activities including antimalarial activity [16,17]. TheTriazine derivative such as cycloguanil and chlorcycloguanil are already approved as antimalarial agents $[18,19]$. In this paper, we used the concept of hybrid molecules $\left(T_{14}-T_{30}\right)$, in which two pharmacophores are linked together to increase their inhibitory activity against covid-19 [20]. Moreover, in order to evaluate their drug-like ability, each proposed compound was evaluated using druglikeness and standard computational pharmacokinetics parameters (ADMET).

The World Health Organization does not recommend or approve any drug for the treatment of covid-19. However, several studies have shown that Hydroxychloroquine $\left(X_{1}\right)$ and Azithromycin $\left(X_{2}\right)$ are a good treatment for COVID-19 [21-23]. In this study, the results of molecular docking of the $X_{1}$ and $X_{2}$ compounds were used as a reference, to determine the required modes of interaction and the key amino acids in the active site of COVID-19 protein protease. Fig. 2 presents the structures $X_{1}$ and $X_{2}$.

\section{Methodology}

\subsection{Docking molecular}

In order to analyze potential binding sites and to better understand the key structural requirements for the compounds under investigation, we performed a molecular docking simulation in silico using AutoDock software with COVID-19 Principal Protease which has been complexed with an N3 inhibitor was solved (PDB ID: 6LU7) [24], obtained from the RCSB Protein Database [25]. First, we used Discovery Studio software [26] to remove the ligands and all the water molecules from the protein. The analysis of ligand-receptor interactions was performed in AutoDock Tools version 1.5.6 [27]. The 3D grid was created using the AUTOGRID algorithm to evaluate the ligand-receptor interaction energy [28]. The grid box was generated with a resolution of $60 \AA$ in all directions $(X$, $\mathrm{Y}, \mathrm{Z}$ axes), the center grid coordinates are $(\mathrm{x}=-15.579 \AA, \mathrm{y}=13.337 \AA$ and $\mathrm{z}=75.418 \AA$ ) and the default grid space size is $0.375 \AA$ for the ligand location in the receptor. The results of the established interactions were graphically presented using Discovery Studio software. Fig. 3 presents the visualization of the interaction modes of crystallized ligand with the active site of the covid-19 protein protease.

\subsection{In silico pharmacokinetics ADMET and drug likeness prediction}

The use of molecular modelling techniques to identify new drug candidates helps to reduce the duration of experimental studies and improve the success rate. For this reason, standard computational pharmacokinetics parameters (ADMET) and drug likeness was established for the preliminary estimation of the physicochemical, pharmacokinetic and drug-like parameters in the drug discovery process. In silico study provides a pathway to access pharmacokinetic parameters (Adsorption, Distribution, Metabolism, Excretion and Toxicity; ADMET) $[29,30]$ Its absorption is the passage of a drug from its site of administration into the systemic circulation, distribution is the diffusion of the drug into the different tissues of the body, Metabolism refers to the chemical 
biotransformation of a drug by the body, Excretion is the elimination of a drug from the body and the toxicity levels of the molecules. Prediction of the drug likeness of the designed compound was estimated by rule-based filters from Lipinski, Ghose, Veber, Egan and Muegge [30], and the synthetic accessibility difficulty scale was 1 to 10 .

\section{Results And Discussion}

\subsection{Molecular docking study}

The interaction modes obtained by molecular docking for compounds $X_{1}$ and $X_{2}$ with covid-19 protein protease are illustrated in Fig. 4 . In this research, we focused only on hydrogen bonding interactions, although the other interactions help to stabilize the ligand inside the active site, because we want to give clear criteria for the choice of active molecules, based on the interactions of the compound $X_{1}$ and $X_{2}$ with the active site, which was a reference for other molecules.

The result of the molecular docking of compound $X_{1}$ with the active site of protein protease shows four interactions through hydrogen bonding with residues

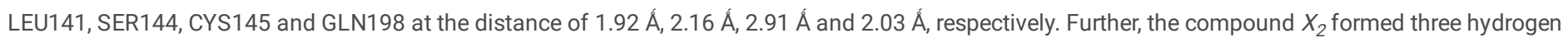
bonding interactions with residues LEU141, GLU143 and SER144 at the distance of $1.81 \AA \AA, 2.75 \AA$, and $2.47 \AA$, respectively. So, compound $X_{1}$ formed four hydrogen bonding interactions and compound $X_{2}$ formed three hydrogen bonding interactions with the SARS-CoV-2 active site. In this study, we consider compounds that have established three or four hydrogen bonding interactions with the SARS-CoV-2 active site as inhibitors of covid-19, and compounds that establish more than four hydrogen bonds are the most effective at fighting this new outbreak. 2D visualization of the interaction modes obtained by molecular docking for compounds $T_{1}-T_{30}$ are illustrated in Fig.5.

All molecules can act as inhibitors of SARS-CoV-2, because they have established many interactions with the active site of covid-19, but with different inhibitory efficacy. Compared to Hydroxychloroquine and azithromycin, most of these molecules $\left(T_{3}, T_{4}, T_{6}, T_{8}, T_{9}, T_{6}, T_{13}, T_{14}, T_{15}, T_{18}, T_{19}, T_{20}, T_{21}, T_{22}, T_{23}\right.$, $T_{26}, T_{28}, T_{30}$ ) have the same efficacy, since they can could form three or four hydrogen bonds with the active site of covid-19. Moreover, some of these molecules $\left(T_{1}, T_{5}, T_{12}, T_{25}, T_{29}\right)$ have shown a higher activity against SARS-CoV-2, because they can form more than four hydrogen bonds with the active site of the covid-19 protein protease.

Based on these results, these molecules can form several hydrogen bonds with the key residues, are therefore theoretically able to bind to the pocket formed by these amino acids of the covid-19 proteins and interfere with the function of SARS-CoV-2. This indicates the potential of these molecules as inhibitors for coronaviruses with similar binding sites and pocket structures. Furthermore, molecules based on quinoline, triazine and azaaurone have shown a great significant anti-SARS-CoV-2 activity in silico, thus it is able to treat patients suffering from Covid 19. In order to increase the effectiveness of these pharmacophores, we have used hybrid molecules. The hybrid molecules such as Quinoline-Oxalamide, Quinoline-Triazine and Azaaurone- Triazine have shown good inhibitory activity of SARS-CoV-2. These pharmacophores could be the shortest path for the development of the drug against the covid-19 virus.

\subsection{ADMET prediction and druglikeness}

In order to ensure that all molecules likely to have anti-SARS-CoV-2 activity could be viable drugs. We used the pharmacokinetic parameters ADMET and Druglikeness. The online tool pkCSM [31] was used to predict the in silico properties of ADMET (Table 1). The Druglikeness properties were also predicted using the SwissADME online tool [32] (Table 2). An absorption value below 30\% indicates poor absorbance [33], the all designed compounds displayed a value greater than $60 \%$ which shows good absorbency in the human intestine. Volume of distribution (VDss) is estimated high if the value is greater than 0.45 . blood brain barrier (BBB) and central nervous system (CNS) permeability standard values ( $>0.3$ to $<-1$ Log BB and $>-2$ to $<-3$ LogPS), respectively. For a given compound a LogBB $<-1$ are poor distributed to brain, while LogBB $>0.3$ are potential to cross BBB and LogPS $>-2$ considered to penetrate the CNS, while LogPS $<-3$ are difficult to move in the CNS [34]. The metabolism refers to the chemical biotransformation of a drug by the body. As a result, drugs produce several metabolites, which may have different physicochemical and pharmacological properties. It is necessary to consider their metabolism of drugs and drug-drug interactions, but there are no sufficient studies or effective drugs to provide information to identify metabolism. A low total clearance value means a decrease in the concentration of the drug in the body, Therefore a high rate of elimination of the drug by the body. Finally, it is required to test whether the predicted compounds are non-toxic, as this plays a key role in drug selection.

According to these criteria, the designed molecules $\left(T_{3}, T_{5}, T_{6}, T_{9}, T_{12}, T_{14}, T_{18}, T_{21}, T_{22}, T_{23}, T_{24}, T_{25}, T_{26}\right)$ have the right pharmacokinetic properties. Based on Drug likeness results, the compounds $\left(T_{1}-T_{15}, T_{19}-T_{26}, T_{29}, T_{30}\right.$ ) respect all drug similarity rules and synthetic accessibility values for all predicted compounds are less than 4 , therefore they are easy to synthesize. Overall, Compounds $\left(T_{3}, T_{5}, T_{6}, T_{9}, T_{12}, T_{14}, T_{21}, T_{22}, T_{23}, T_{24}, T_{25}, T_{26}\right)$ have the potential to become excellent drug candidates and are easy to synthesize. As these results appear very promising, it should be recalled that these results were obtained only by in silico predictions, further bioassays and clinical trials are needed to confirm the inhibitory activity of these candidate drugs against SARS-Cov- 2 .

\section{Conclusion}

In this study, we succeeded in determining the key amino acids and the number of hydrogen bonds required to select compounds with inhibitory potential for this new coronavirus, based on hydroxychloroquine and azithromycin drugs as reference. The orientation of researchers towards a new research direction aimed at testing molecules based on quinoline, triazine and azaaurone and the use of hybrid molecules such as quinoline-oxalamide, quinoline-triazine and azaaurone-triazine could be the shortest path to produce drugs against this new outbreak, because these molecules have shown high inhibitory efficacy and promising results in silico ADMET evaluations and drug likeness. We hope that this work could contribute to global efforts to develop effective drugs for SARS-CoV-2. 


\section{Declarations}

Funding statement

This research did not receive any specific grant from funding agencies in the public, commercial, or not-for-profit sectors.

Competing interest statement

The authors declare that there are no conflicts of interest regarding the publication of this paper.

Additional information

No additional information is available for this paper.

\section{References}

[1]D. Cavanagh, Coronavirus avian infectious bronchitis virus, Vet. Res. 38 (2007) 281-297. https://doi.org/10.1051/vetres:2006055

[2]S. R. Weiss, J. L. Leibowitz, Coronavirus pathogenesis, in: Adv. Virus Res., Academic Press Inc., 2011: pp. 85-164. https://doi.org/10.1016/B978-0-12385885-6.00009-2.

[3]Y. Lim, Y. Ng, J. Tam, D. Liu, Human Coronaviruses: A Review of Virus-Host Interactions, Diseases. 4 (2016) 26. https://doi.org/10.3390/diseases4030026.

[4]K. Dhama, R. V. S. Pawaiya, S. Chakraborty, R. Tiwari, M. Saminathan, A. K. Yerma, Coronavirus infection in equines: A review, Asian J. Anim. Vet. Adv. 9 (2014) 164-176. https://doi.org/10.3923/ajava.2014.164.176.

[5]J. Cui, F. Li, Z. L. Shi, Origin and evolution of pathogenic coronaviruses, Nat. Rev. Microbiol. 17 (2019) 181-192. https://doi.org/10.1038/s41579-0180118-9.

[6]WHO | Summary of probable SARS cases with onset of illness from 1 November 2002 to 31 July 2003, WHO. (2015).

[7]WHO | Middle East respiratory syndrome coronavirus (MERS-CoV), (n.d.). https://www.who.int/emergencies/mers-cov/en/ (accessed April 17, 2020).

[8]J. F.-W. Chan, K.-H. Kok, Z. Zhu, H. Chu, K. K.-W. To, S. Yuan, K.-Y. Yuen, Genomic characterization of the 2019 novel human-pathogenic coronavirus isolated from a patient with atypical pneumonia after visiting Wuhan, Emerg. Microbes Infect. 9 (2020) 221-236. https://doi.org/10.1080/22221751.2020.1719902.

[9]D. S. Hui, E. I Azhar, T. A. Madani, F. Ntoumi, R. Kock, O. Dar, G. Ippolito, T. D. Mchugh, Z. A. Memish, C. Drosten, A. Zumla, E. Petersen, The continuing 2019nCoV epidemic threat of novel coronaviruses to global health-The latest 2019 novel coronavirus outbreak in Wuhan, China, Int. J. Infect. Dis. 91 (2020) 264266. https://doi.org/10.1016/j.ijid.2020.01.009.

[10]C. C. Lai, T. P. Shih, W. C. Ko, H. J. Tang, P. R. Hsueh, Severe acute respiratory syndrome coronavirus 2 (SARS-CoV-2) and coronavirus disease-2019 (COVID-19): The epidemic and the challenges, Int. J. Antimicrob. Agents. 55 (2020) 105924. https://doi.org/10.1016/j.ijantimicag.2020.105924.

[11]N. Oliver, X. Barber, K. Roomp, K. Roomp, The Covid19Impact Survey: Assessing the Pulse of the COVID-19 Pandemic in Spain via 24 questions, (2020).

[12]S. P. Adhikari, S. Meng, Y. J. Wu, Y. P. Mao, R. X. Ye, Q. Z. Wang, C. Sun, S. Sylvia, S. Rozelle, H. Raat, H. Zhou, Epidemiology, causes, clinical manifestation and diagnosis, prevention and control of coronavirus disease (COVID-19) during the early outbreak period: A scoping review, Infect. Dis. Poverty. 9 (2020) 112. https://doi.org/10.1186/s40249-020-00646-x.

[13]S. Sarvagalla, S. B. Syed, M. S. Coumar, An Overview of Computational Methods, Tools, Servers, and Databases for Drug Repurposing, in: Silico Drug Des., Elsevier, 2019: pp. 743-780. https://doi.org/10.1016/b978-0-12-816125-8.00025-0.

[14]H. Hadni, M. Mazigh, M. Elhallaoui, QSAR and Molecular docking studies of 4-anilinoquinoline-triazine hybrids as pf-DHFR inhibitors, 8 (2019) 84-93.

[15]H. Hadni, M. Elhallaoui, Molecular docking and QSAR studies for modeling the antimalarial activity of hybrids 4-anilinoquinoline-triazines derivatives with the wild-type and mutant receptor pf-DHFR, Heliyon. 5 (2019) e02357. https://doi.org/10.1016/j.heliyon.2019.e02357.

[16] hanine hadni, menana elhallaoui, 2D and 3D-QSAR, molecular docking and ADMET properties in silico studies of azaaurones as antimalarial agents, New J. Chem. (2020). https://doi.org/10.1039/c9nj05767f.

[17]H. Hadni, M. Elhallaoui, 3D-QSAR, docking and ADMET properties of aurone analogues as antimalarial agents, Heliyon. 6 (2020) e03580. https://doi.org/10.1016/j.heliyon.2020.e03580.

[18]M. D. Hastings, C. H. Sibley, Pyrimethamine and WR99210 exert opposing selection on dihydrofolate reductase from Plasmodium vivax, Proc. Natl. Acad. Sci. 99 (2002) 13137-13141. https://doi.org/10.1073/pnas.182295999.

[19]H. Hadni, M. Mazigh, E. Charif, A. Bouayad, M. Elhallaoui, Molecular Modeling of Antimalarial Agents by 3D-QSAR Study and Molecular Docking of Two Hybrids 4-Aminoquinoline-1,3,5-triazine and 4-Aminoquinoline-oxalamide Derivatives with the Receptor Protein in Its Both Wild and Mutant Types, Biochem. 
Res. Int. 2018 (2018) 1-15. https://doi.org/10.1155/2018/8639173.

[20]F. W. Muregi, A. Ishih, Next-generation antimalarial drugs: hybrid molecules as a new strategy in drug design, Drug Dev. Res. 71 (2009) n/a-n/a. https://doi.org/10.1002/ddr.20345.

[21]P. Gautret, J.-C. Lagier, P. Parola, V. T. Hoang, L. Meddeb, M. Mailhe, B. Doudier, J. Courjon, V. Giordanengo, V. E. Vieira, H. T. Dupont, S. Honoré, P. Colson, E. Chabrière, B. La Scola, J.-M. Rolain, P. Brouqui, D. Raoult, Hydroxychloroquine and azithromycin as a treatment of COVID-19: results of an open-label nonrandomized clinical trial, Int. J. Antimicrob. Agents. (2020) 105949. https://doi.org/10.1016/j.ijantimicag.2020.105949.

[22]J. Gabriels, M. Saleh, D. Chang, L. M. Epstein, Inpatient Use of Mobile Continuous Telemetry for COVID-19 Patients Treated with Hydroxychloroquine and Azithromycin, Hear. Case Reports. 0 (2020). https://doi.org/10.1016/j.hrcr.2020.03.017.

[23]A. K. Singh, A. Singh, A. Shaikh, R. Singh, A. Misra, Chloroquine and hydroxychloroquine in the treatment of COVID-19 with or without diabetes: A systematic search and a narrative review with a special reference to India and other developing countries, Diabetes Metab. Syndr. Clin. Res. Rev. 14 (2020) 241-246. https://doi.org/10.1016/j.dsx.2020.03.011.

[24]Z. Jin, X. Du, Y. Xu, Y. Deng, M. Liu, Y. Zhao, B. Zhang, X. Li, L. Zhang, C. Peng, Y. Duan, J. Yu, L. Wang, K. Yang, F. Liu, R. Jiang, X. Yang, T. You, X. Liu, X. Yang, F. Bai, H. Liu, X. Liu, L. W. Guddat, W. Xu, G. Xiao, C. Qin, Z. Shi, H. Jiang, Z. Rao, H. Yang, Structure of Mpro from COVID-19 virus and discovery of its inhibitors, BioRxiv. (2020) 2020.02.26.964882. https://doi.org/10.1101/2020.02.26.964882.

[25]A. Kouranov, L. Xie, J. de la Cruz, L. Chen, J. Westbrook, P. E. Bourne, H. M. Berman, The RCSB PDB information portal for structural genomics, Nucleic Acids Res. 34 (2006) D302-D305. https://doi.org/10.1093/nar/gkj120.

[26]D. S. BIOvIA, Discovery studio modeling environment., San Diego, Dassault Syst. Release, 4. (2015). https://doi.org/https://doi.org/10.11436/mssj.17.98.

[27]G. M. Morris, R. Huey, W. Lindstrom, M. F. Sanner, R. K. Belew, D. S. Goodsell, A. J. Olson, AutoDock4 and AutoDockTools4: Automated docking with selective receptor flexibility, J. Comput. Chem. 30 (2009) 2785-2791. https://doi.org/10.1002/jcc.21256.

[28]G. M. Morris, D. S. Goodsell, R. S. Halliday, R. Huey, W. E. Hart, R. K. Belew, A. J. Olson, Automated docking using a Lamarckian genetic algorithm and an empirical binding free energy function, J. Comput. Chem. 19 (1998) 1639-1662. https://doi.org/10.1002/(SICI)1096-987X(19981115)19:14<1639::AIDJCC10>3.0.CO;2-B.

[29]L. L. G. Ferreira, A.D. Andricopulo, ADMET modeling approaches in drug discovery, Drug Discov. Today. 24 (2019) 1157-1165.

https://doi.org/10.1016/j.drudis.2019.03.015.

[30]C. Y. Jia, J. Y. Li, G. F. Hao, G. F. Yang, A drug-likeness toolbox facilitates ADMET study in drug discovery, Drug Discov. Today. 25 (2020) $248-258$.

https://doi.org/10.1016/j.drudis.2019.10.014.

[31]D. E. V. Pires, T. L. Blundell, D. B. Ascher, pkCSM: Predicting Small-Molecule Pharmacokinetic and Toxicity Properties Using Graph-Based Signatures, J. Med. Chem. 58 (2015) 4066-4072. https://doi.org/10.1021/acs.jmedchem.5b00104.

[32]A. Daina, O. Michielin, V. Zoete, SwissADME: A free web tool to evaluate pharmacokinetics, drug-likeness and medicinal chemistry friendliness of small molecules, Sci. Rep. 7 (2017). https://doi.org/10.1038/srep42717.

[33]W. J. Egan, K. M. Merz, J. J. Baldwin, Prediction of drug absorption using multivariate statistics, J. Med. Chem. 43 (2000) $3867-3877$. https://doi.org/10.1021/jm000292e.

[34]D. E. Clark, In silico prediction of blood-brain barrier permeation, Drug Discov. Today. 8 (2003) 927-933. https://doi.org/10.1016/S13596446(03)02827-7.

\section{Tables}

Table 1. In silico ADMET prediction of new designed compounds $\left(\mathrm{T}_{1}-\mathrm{T}_{\mathbf{3 0}}\right)$. 


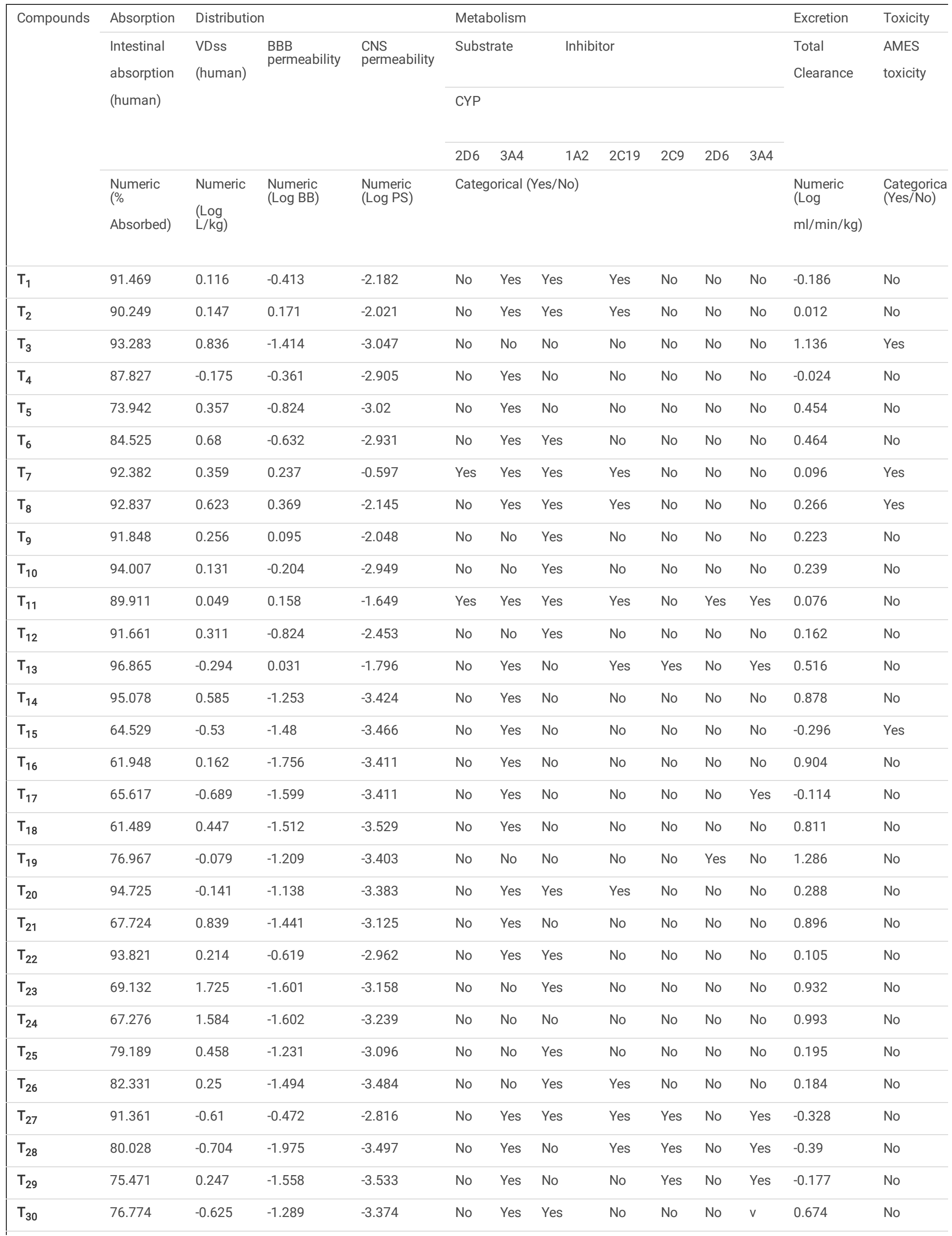


Table 2. Drug likeness prediction of the compounds $\left(T_{1}-T_{30}\right)$ basing on lipinski, Ghose ,veber, Egan and Muegge, and their synthetic accessibility.

\begin{tabular}{|c|c|c|c|c|c|c|}
\hline \multirow[t]{2}{*}{ Compounds } & \multicolumn{6}{|c|}{ Druglikeness } \\
\hline & Lipinski & Ghose & Veber & Egan & Muegge & Synthetic accessibility \\
\hline $\mathrm{T}_{1}$ & Yes & Yes & Yes & Yes & Yes & 3.04 \\
\hline $\mathrm{T}_{2}$ & Yes & Yes & Yes & Yes & Yes & 2.09 \\
\hline $\mathrm{T}_{3}$ & Yes & Yes & Yes & Yes & Yes & 2.32 \\
\hline $\mathrm{T}_{4}$ & Yes & Yes & Yes & Yes & Yes & 3.40 \\
\hline $\mathrm{T}_{5}$ & Yes & Yes & Yes & Yes & Yes & 4.12 \\
\hline $\mathrm{T}_{6}$ & Yes & Yes & Yes & Yes & Yes & 3.95 \\
\hline $\mathrm{T}_{7}$ & Yes & Yes & Yes & Yes & Yes & 2.50 \\
\hline $\mathrm{T}_{8}$ & Yes & Yes & Yes & Yes & Yes & 2.66 \\
\hline$T_{9}$ & Yes & Yes & Yes & Yes & Yes & 2.61 \\
\hline $\mathrm{T}_{10}$ & Yes & Yes & Yes & Yes & Yes & 2.88 \\
\hline $\mathrm{T}_{11}$ & Yes & Yes & Yes & Yes & Yes & 2.94 \\
\hline $\mathrm{T}_{12}$ & Yes & Yes & Yes & Yes & Yes & 2.92 \\
\hline $\mathrm{T}_{13}$ & Yes & Yes & Yes & Yes & Yes & 3.68 \\
\hline $\mathrm{T}_{14}$ & Yes & Yes & No & Yes & Yes & 2.58 \\
\hline $\mathrm{T}_{15}$ & Yes & Yes & Yes & Yes & Yes & 2.94 \\
\hline $\mathrm{T}_{16}$ & Yes & Yes & No & Yes & Yes & 3.83 \\
\hline $\mathrm{T}_{17}$ & Yes & Yes & No & Yes & Yes & 3.10 \\
\hline $\mathrm{T}_{18}$ & Yes & Yes & No & Yes & Yes & 3.50 \\
\hline $\mathrm{T}_{19}$ & Yes & Yes & Yes & Yes & Yes & 2.47 \\
\hline $\mathrm{T}_{20}$ & Yes & Yes & Yes & Yes & Yes & 2.17 \\
\hline $\mathrm{T}_{21}$ & Yes & Yes & Yes & Yes & Yes & 2.47 \\
\hline $\mathrm{T}_{22}$ & Yes & Yes & Yes & Yes & Yes & 2.61 \\
\hline $\mathrm{T}_{23}$ & Yes & Yes & Yes & Yes & Yes & 3.2 \\
\hline$T_{24}$ & Yes & Yes & Yes & Yes & Yes & 3.19 \\
\hline $\mathrm{T}_{25}$ & Yes & Yes & Yes & Yes & Yes & 2.6 \\
\hline$T_{26}$ & Yes & Yes & Yes & Yes & Yes & 3.59 \\
\hline$T_{27}$ & Yes & Yes & Yes & Yes & No & 3.37 \\
\hline $\mathrm{T}_{28}$ & Yes & Yes & Yes & Yes & No & 3.63 \\
\hline$T_{29}$ & Yes & Yes & Yes & Yes & Yes & 3.66 \\
\hline$T_{30}$ & Yes & Yes & Yes & Yes & Yes & 3.49 \\
\hline
\end{tabular}


Figures

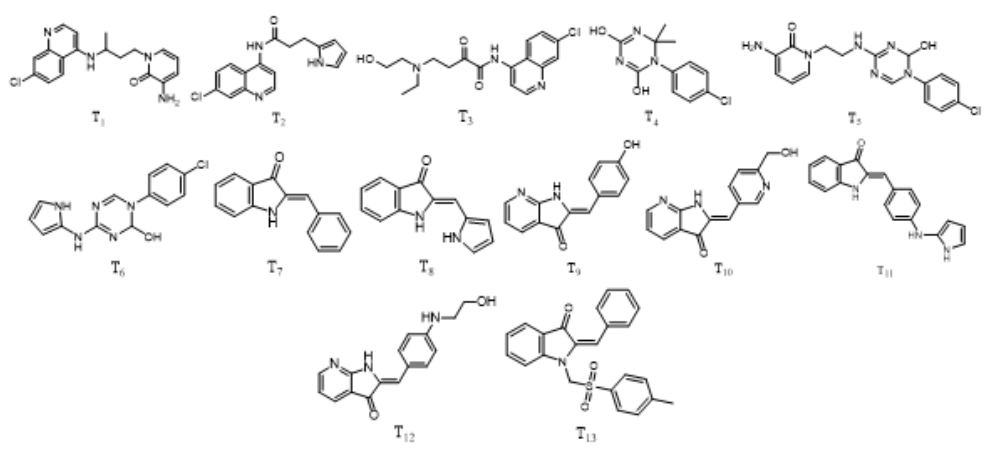

rath"

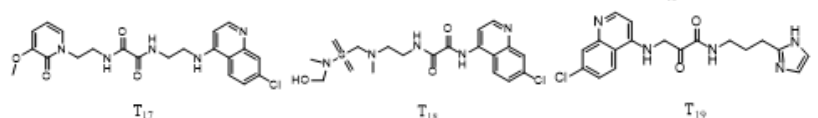
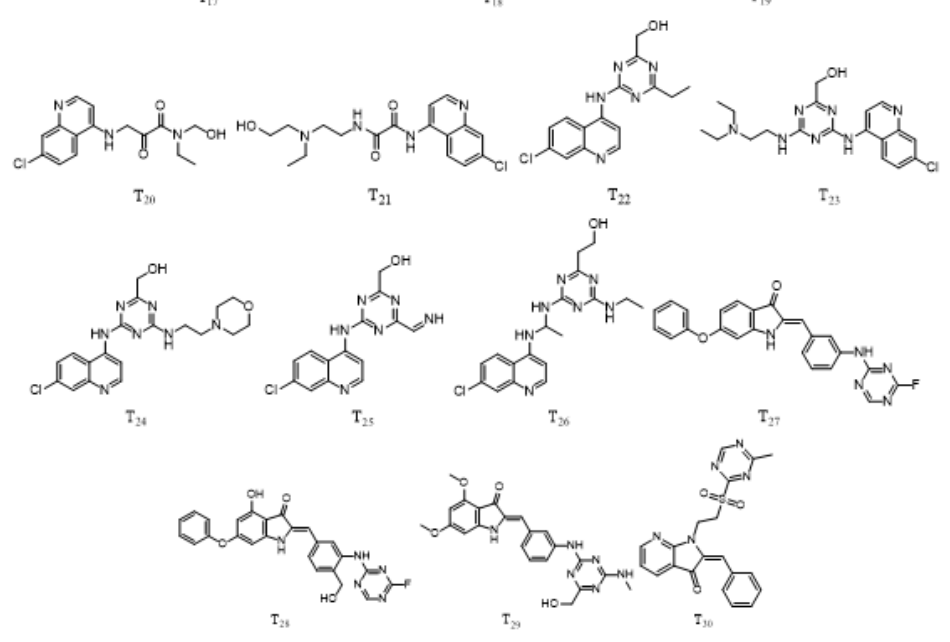

Figure 1

All the molecules used in molecular docking<smiles>CCN(CCO)CCCC(C)Nc1ccnc2cc(Cl)ccc12</smiles>

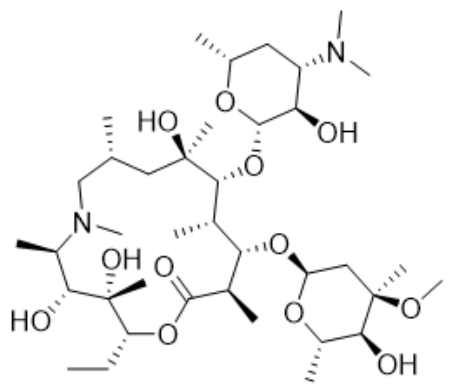

$\mathrm{X}_{1}$

$\mathrm{X}_{2}$

Figure 2

Structure of hydroxychloroquine (X1) and azithromycin (X2)

Page $8 / 10$ 


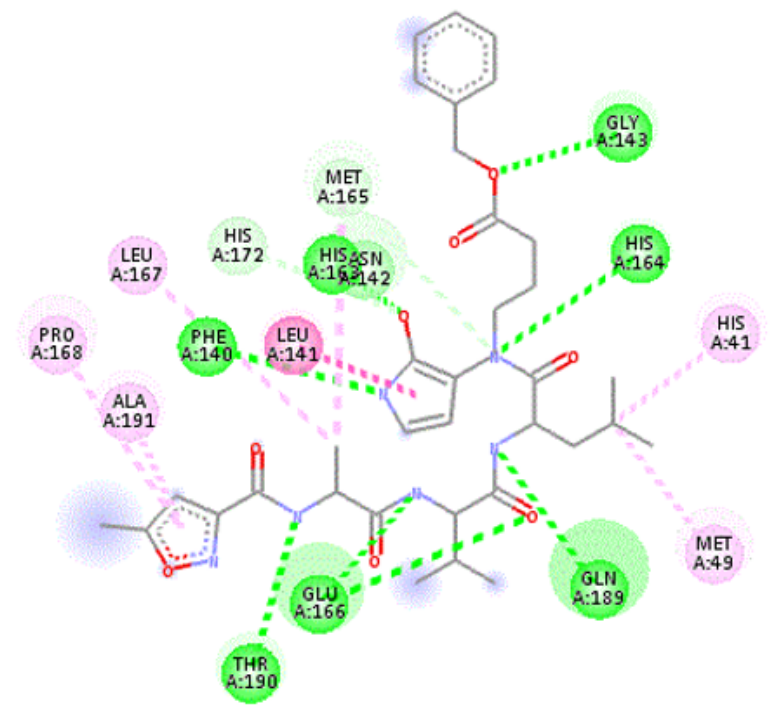

\section{Interactions}

van der Waals

Conventional Hydrogen Bond

Carbon Hydrogen Bond

Amide-Pi Stacked

Alkyl

Pi-Alkyl

Figure 3

2D visualization showing interactions of the crystallographic ligand pose with the SARS-CoV-2 protein protease.

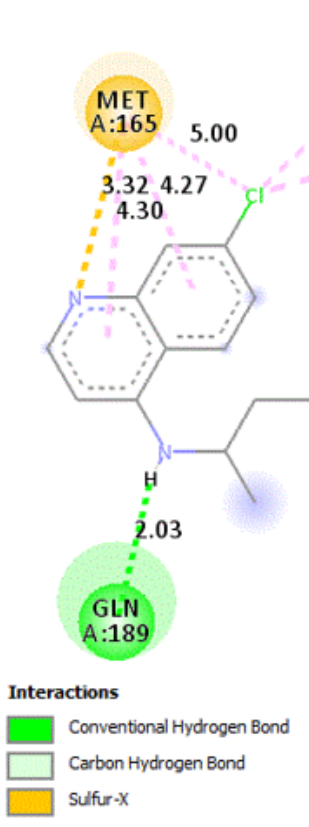

$\mathbf{X}_{\mathbf{1}}$

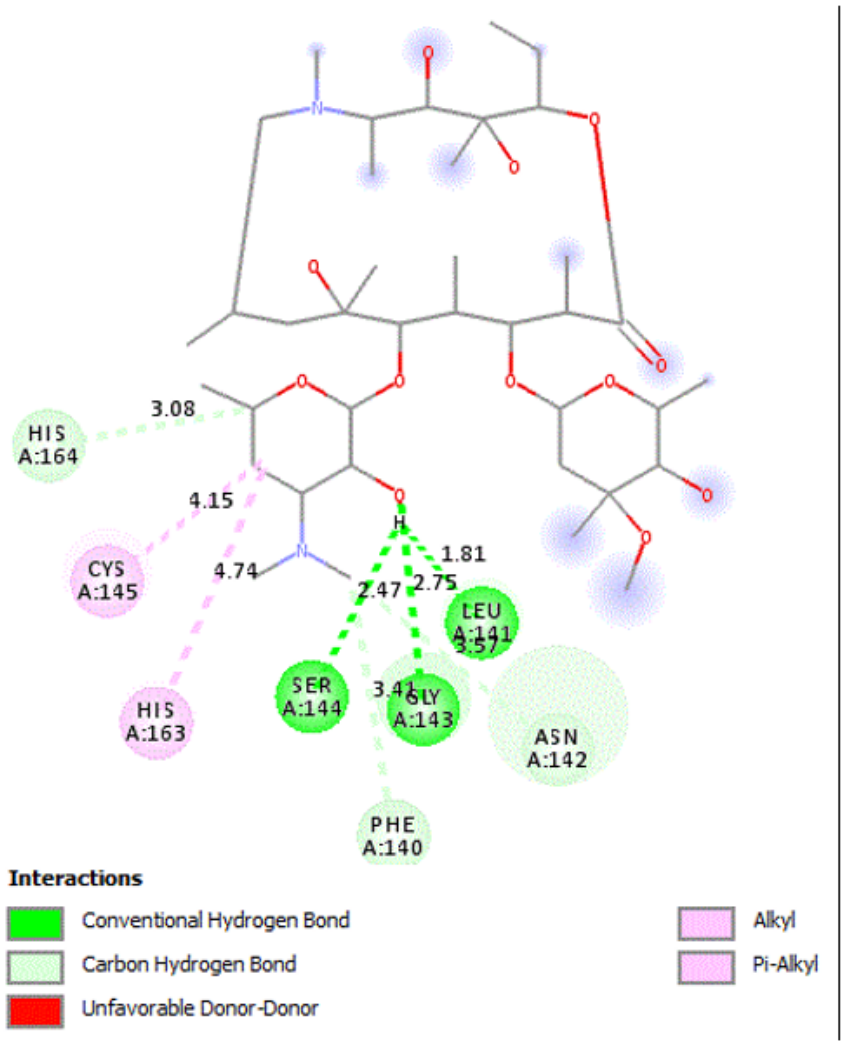

$\mathbf{X}_{2}$

\section{Figure 4}

2D docking poses showing interactions of compounds $\mathrm{X} 1$ and $\mathrm{X} 2$ in the binding sites of SARS-CoV-2 protein protease.. (a) Compound X1: (binding energy $-7.51 \mathrm{kcal} / \mathrm{mol}$ ). (b) Compound X2: (binding energy $-6.13 \mathrm{kcal} / \mathrm{mol}$ ) 

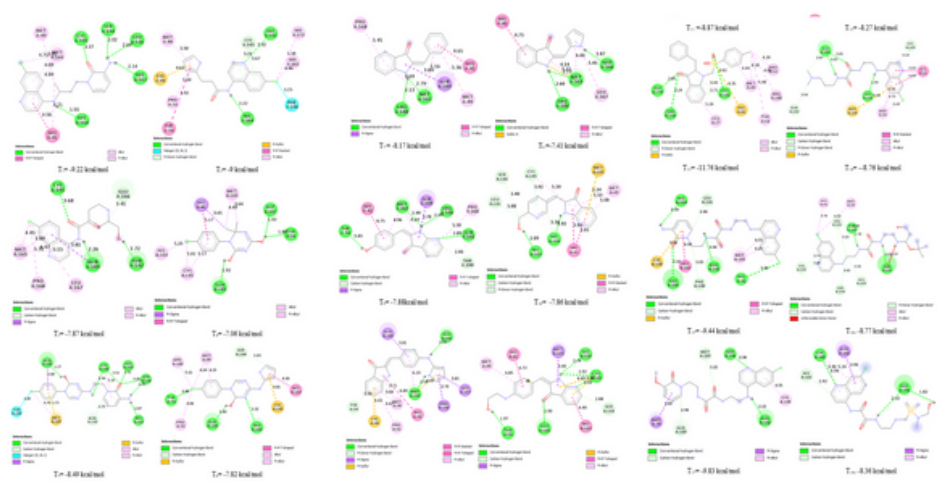

tortinter

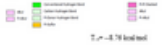
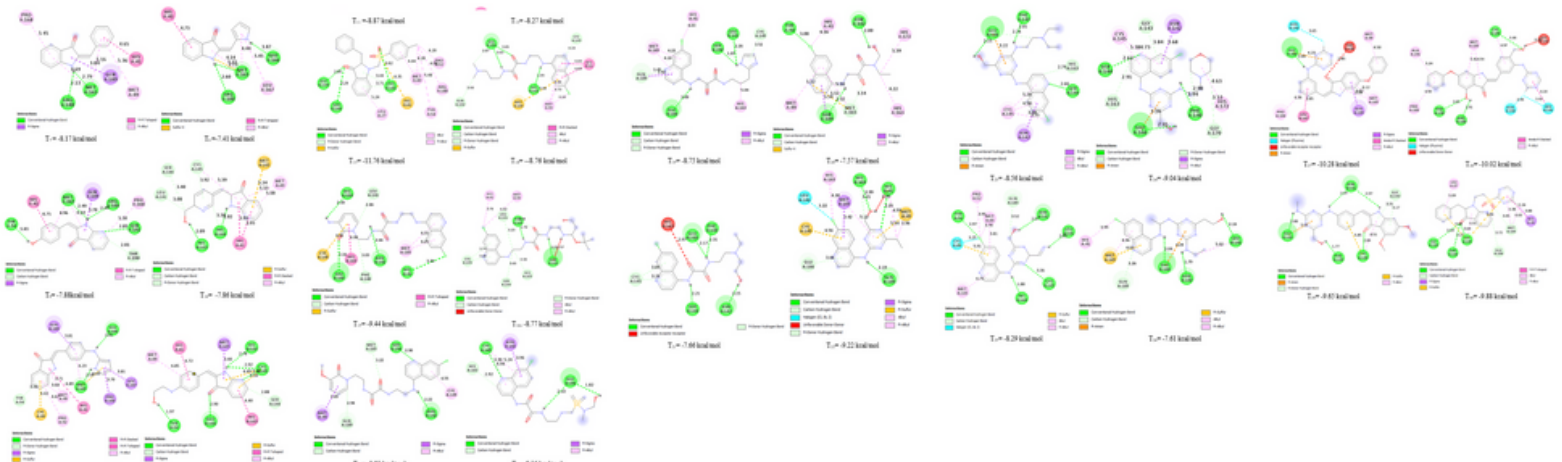
풀 $7=$

\section{Figure 5}

$2 \mathrm{D}$ visualization of the distances and interaction modes with the active site of SARS-CoV-2 protein protease. (Also available in the Supplementary Files section.)

\section{Supplementary Files}

This is a list of supplementary files associated with this preprint. Click to download.

- Figure.docx 